\title{
Der „insertierbare“ Herzmonitor
}

Thorsten Lewalter, Ilia Koutsouraki, Turgut Brodherr

\begin{abstract}
Herzrhythmusstörungen können harmlos sein - aber auch lebensbedrohlich. Gemeinsam ist beiden, dass sie nur sporadisch auftreten und deshalb nicht einfach zu messen sind. Hier hilft ein insertierbarer Herzmonitor: Er kann mehrere Jahre im Körper verbleiben und den Herzrhythmus des Patienten Tag und Nacht überwachen. Erfahren Sie hier, wie die Implantation eines Herzmonitors abläuft.
\end{abstract}

Abb. 1 Gerätpositionierung. Die optimale Position (grün) ist der 4 . ICR $/ 45^{\circ}$, alternativ dazu parallel, parasternal (blau).

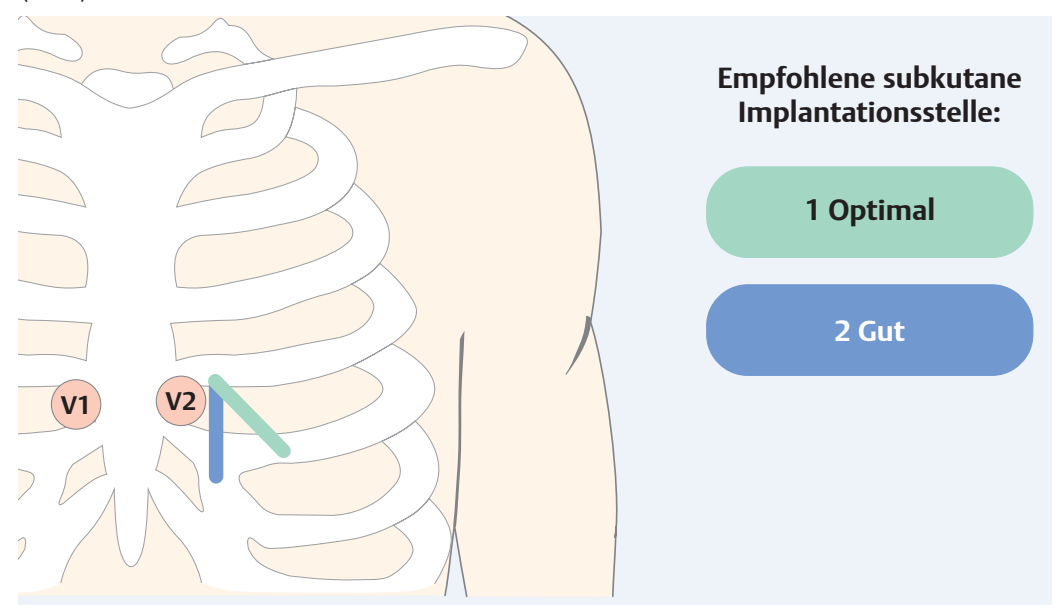

Diagnose von Herzrhythmusstörungen I Der Goldstandard bei der Diagnostik von Herzrhythmusstörungen ist ein Elektrokardiogramm zum Zeitpunkt der Rhythmusstörung. Es gibt Aufschluss über die Art und die Länge der Episode. Außerdem unterstützt es den Arzt bei der Risikostratifizierung der Rhythmusstörung und hilft ihm, die richtige und oft lebensrettende Therapie festzulegen. Es gibt viele verschiedene Herzrhythmusstörungen. Manche sind harmlos, viele sind aber auch lebensbedrohend. Eins haben sie gemeinsam: Sie sind meist nicht permanent, sondern treten anfallsweise auf. Ein herkömmliches 12-Kanal-EKG ist bei der Detektion nicht hilfreich.

Permanente Überwachung | Auch LangzeitholterEKGs von 24 Stunden bis hin zu 7 Tagen erfassen nicht alle Rhythmusstörungen. Deshalb gibt es Herzmonitore zur permanenten Überwachung. Hierbei handelt es sich um ein Implantat, das unter die Haut geschoben wird. Dort kann es mehrere Jahren verbleiben und den Herzrhythmus kontinuierlich als Schleifenmonitor aufzeichnen.

Ursprünglich wurden Herzmonitore entwickelt, um Herzrhythmusstörungen aufzudecken, die zu Synkopen, also kurzeitigen, von allein terminierenden Ohnmachten, führen.
Indikationen | Die Indikationen für die Implantation eines Herzmonitors sind:

- ungeklärte rezidivierende Synkopen mit einem Rezidivintervall größer 4 Wochen [1-3]

- Sekundärprophylaxe ungeklärter Schlaganfälle - Vorhofflimmer-Monitoring nach Ablation [4]

- unklare Palpitationen bei Verdacht auf eine Herzrhythmusstörung [1]

Patientenaufklärung | Der Patient muss 24 Stunden voher mündlich und schriftlich über die Prozedur sowie mögliche Risiken (z. B. Infektion, Blutung oder Geräteabstoßung) und Nebenwirkungen (Wundschmerz etc.) aufgeklärt werden. Spezielle Aufklärungsbögen sind verfügbar.

\section{So wird's gemacht: Implantation des Monitors}

Material | Folgende Materialien werden für den Eingriff benötigt:

- OP-Kittel, sterile Handschuhe, großes Lochtuch

- Schale für Desinfektionsmittel, Hautdesinfektion, Klemme zum Waschen der Haut

- Kanüle, Spritze (10-20 ml), lokales Betäubungsmittel (z. B. Xylocain)

- Kompressen/Tupfer

- Nahtmaterial (Nadelhalter!) oder alternativ Steristrips

- steriles Pflaster für die Wunde

- Reveal-LINQ-Herzmonitor, inklusive Implantationstool und Hautstanzmesser

- Programmiergerät

Modell | Es gibt verschiedene Herzmonitore auf dem Markt. Hier wird der Eingriff anhand des Reveal-LINQ-Herzmonitors erläutert. Das Gerät ist mit 1,2 cm das kleinste verfügbare Device.

Behandlungsraum | Der Herzmonitor wird in einem minimalinvasiven Verfahren mit Hilfe eines „Insertionstools“ unter die Haut geschoben. Dies sollte in einem geeigneten Eingriffs- oder Behandlungsraum geschehen, in dem aseptisches Arbei- 


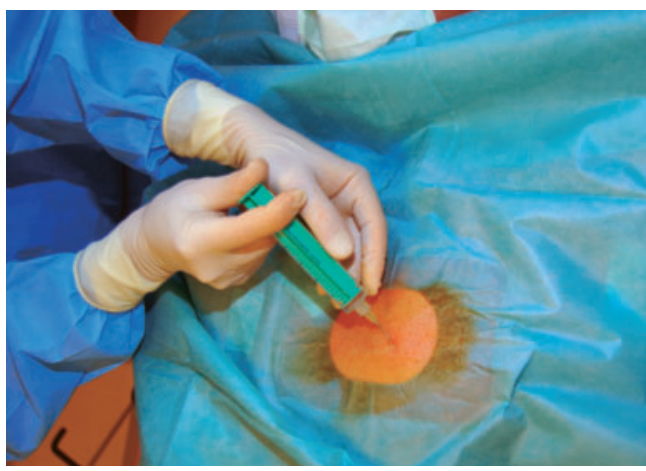

Abb. 2 Der Patient wird abgedeckt und eine lokale Anästhesie gesetzt.

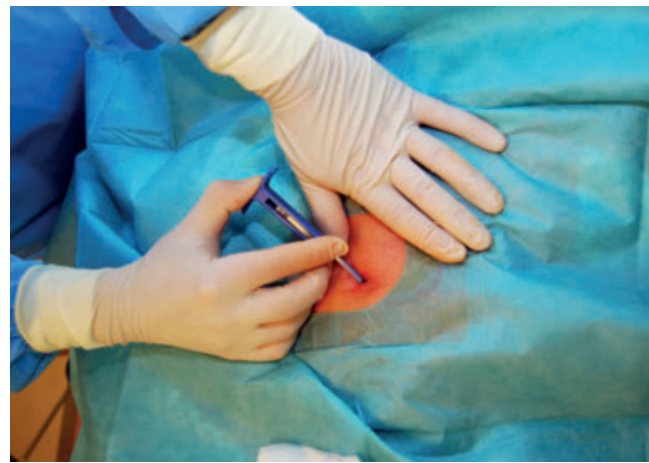

Abb. 4 Der Tunnelierungsfortsatz des Einführbestecks wird in die Subkutis vorgeschoben.

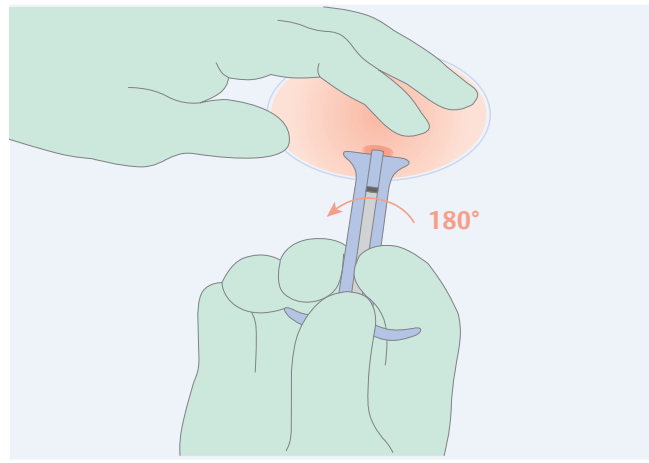

Abb. 6 Nach vollständigem Einführen wird das Einführbesteck um $180^{\circ}$ gedreht.

ten möglich ist. Ein OP oder Herzkatheterlabor ist nicht notwendig.

Tunnelierungstool | Der Anwender sollte im Umgang mit aseptischen Arbeitstechniken wie dem Abwaschen, Abdecken und dem Setzen der Lokalanästhesie vertraut sein. Erfahrung mit invasiven Therapien ist von Vorteil. Eine gute und vollständige Einweisung in das System muss vorab erfolgen. Das Implantationsset enthält ein Tunnelierungstool, das auch als Einführbesteck dient. Der Herzmonitor steckt bereits fertig in dem Tool. Das Set enthält außerdem ein Hautstanzmesser für den Hautschnitt.

Eingabe der Patientendaten I Der Herzmonitor Reveal LINQ wird parasternal links, subkutan pa-

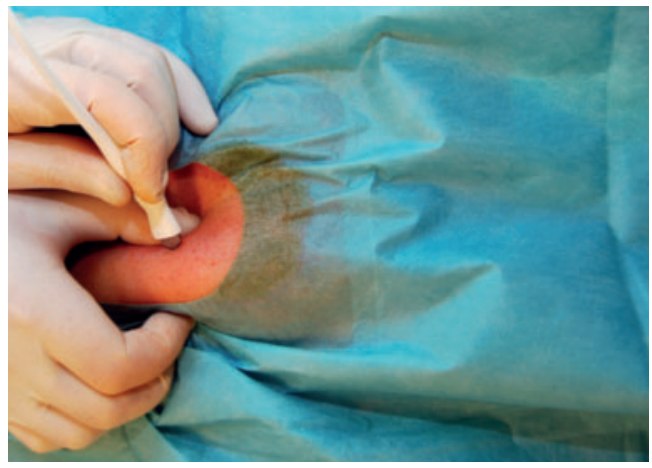

Abb. 3 Mit dem Hautstanzmesser wird in kaudale Richtung eingestochen.

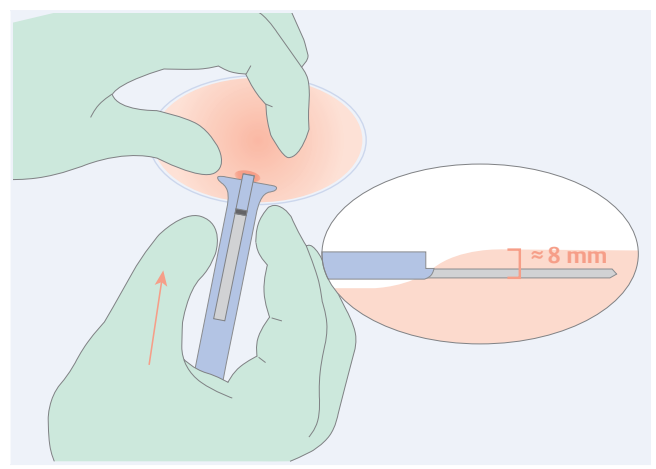

Abb. 5 Hier ist es wichtig, den Griff abzusenken, um dicht unter der Hautoberfläche (ca. $8 \mathrm{~mm}$ ) zu bleiben.

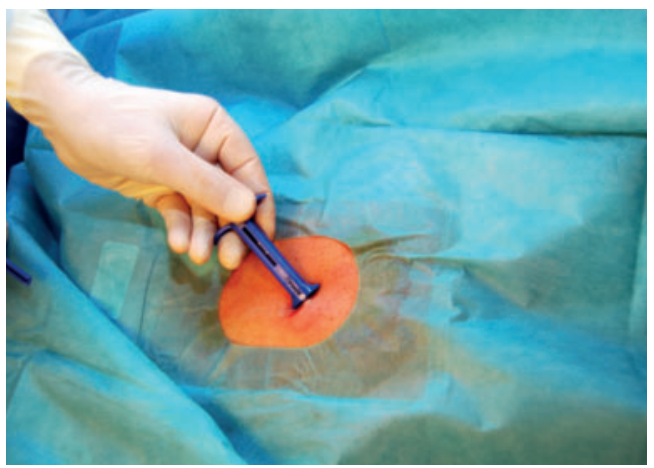

Abb. 7 Nach dem Drehen des Tunnelierungstools.

rallel zur Hautoberfläche etwa $8 \mathrm{~mm}$ tief unter der Haut platziert. Vor der Prozedur werden die Patientendaten mit Hilfe des Programmiergeräts im Implantat gespeichert. Hierbei ist auch die Indikation (der Überwachungsgrund) einzugeben. Automatisch wird der kardiale Monitor so für den jeweiligen Anwendungsfall konfiguriert. Dazu zählen:

- Synkope

- Palpitation

- Schlaganfall

- AF-Monitoring

Position markieren I Der Patient befindet sich mit angelegten Armen in Rückenlage. Die GerätePosition wird mit einem Stift markiert. Die optimale Position ist der 4 . ICR $/ 45^{\circ}(\triangleright$ Abb. 1 [grün]), 


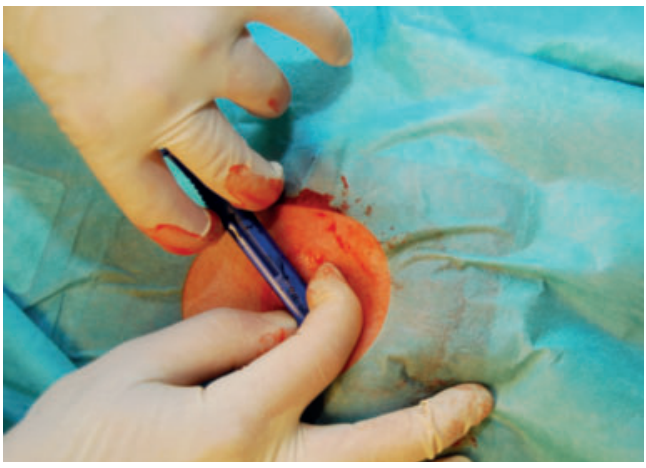

Abb. 8 Vorschieben des Kolbens. Die freie Hand des Anwenders sichert die Position des Einführbestecks.

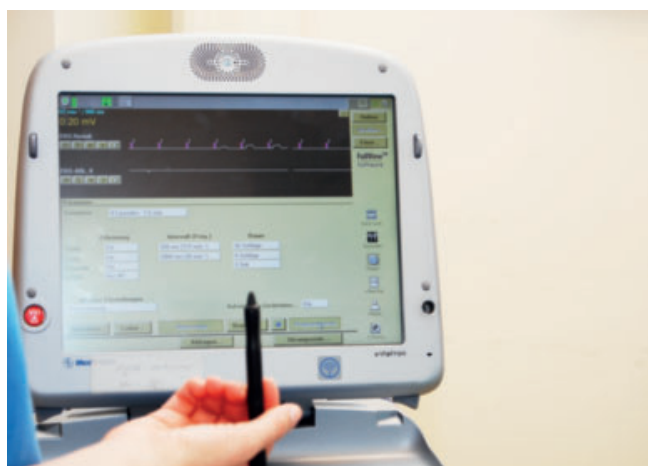

Abb. 10 Mit dem Programmiergerät 2090 wird die Signalstärke des Implantats überprüft.

alternativ dazu parallel, parasternal $(\bullet$ Abb. 1 [blau]). Die Eingriffsstelle wird so abgewaschen, wie es bei einem operativen Eingriff üblich ist. Nachdem der Arzt Kittel und Handschuhe angelegt hat, wird der Patient abgedeckt und die lokale Anästhesie gesetzt ( $\bullet$ Abb. 2).

Einführungsprozedur | Der Anwender positioniert sich rechts vom Patienten auf Höhe der Schulter. Dies ermöglicht eine Arbeitsweise in Richtung der Herzachse. Es wird eine Hautfalte gebildet und das Hautstanzmessers in kaudale Richtung eingestochen ( $\bullet$ Abb. 3). Der Tunnelierungsfortsatz des Einführbestecks wird nun in Richtung der Position parallel zur Hautoberfläche in die Subkutis vorgeschoben.

Hier ist es wichtig, den Griff abzusenken, um dicht unter der Hautoberfläche (ca. $8 \mathrm{~mm}$ ) zu bleiben ( $\bullet$ Abb. 4 und 5 )

Nach vollständigem Einführen wird das Einführbesteck um $180^{\circ}$ gedreht ( Abb. 6 und 7). Nun wird der Kolben aufgesetzt: Die freie Hand des Anwenders sichert die Position des Einführbestecks, damit es nicht zurückweicht, während der Kolben vollständig vorgeschoben wird ( $\bullet$ Abb. 8).

Platzierung des Monitors I Der Herzmonitor muss $10 \mathrm{~mm}$ von der Inzision entfernt platziert werden. Der Kolben wird nun entfernt, wobei die

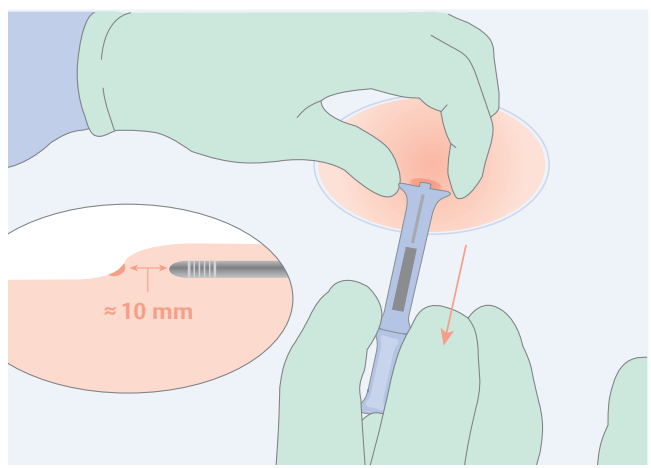

Abb. 9 Zurückziehen des Tunnelierungstools.

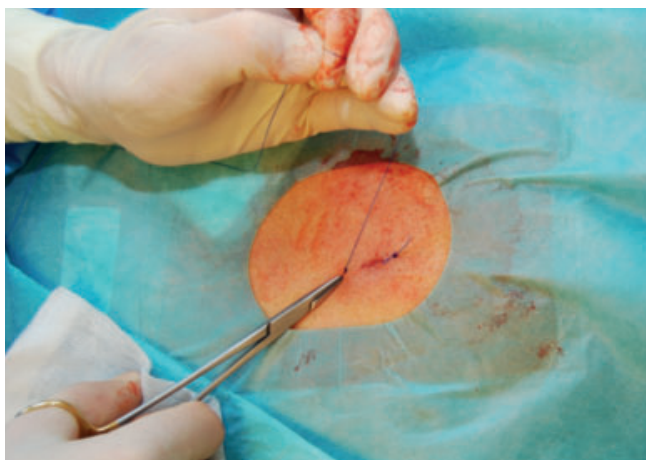

Abb. 11 Die Wunde wird mit einer Hautnaht verschlossen.

freie Hand durch leichten Druck auf die Inzisionsstelle verhindert, dass das Implantat zurückweicht $(\checkmark$ Abb. 9). Danach wird in gleicher Weise das Einführbesteck entfernt. Das Besteck bleibt im sterilen Feld, um es ggf. wiederzuverwenden, falls bei nicht ausreichender Signalstärke eine Repositionierung nötig ist.

Abschluss des Eingriffs | Nach der Kontrolle der Signalstärke (mindestens $>0,20 \mathrm{mV}$ ) mit dem Programmiergerät $2090(\bullet$ Abb. 10) wird die Blutung durch eine anhaltende Kompression gestillt. Im Anschluss wird die Wunde mit einer Hautnaht ( Abb. 11) oder alternativ mit Steristrips verschlossen und mit einem Pflaster abgeklebt.

Nachsorge | Unmittelbar im Anschluss an den Eingriff wird das Implantat mit dem Telemedizinmonitor verbunden. Dieser Patienten-Monitor ist eine Kommunikationseinheit, die auf dem Nachttisch des Patienten platziert wird und jede Nacht automatisch die Episodendaten des Implantats via Mobilfunknetz zu einem Internetserver sendet. Der Anwender kann das System so konfigurieren, dass er nur bei ausgewählten RhythmusEreignissen benachrichtigt wird ( $\bullet$ Abb. 12). Dem Arzt steht somit ein komplettes System zum kontinuierlichen Monitoring und eine sehr zeitnahe Datenauswertung zur Verfügung.

Kostenerstattung | Im Rahmen eines stationären Aufenthalts ist eine Vergütung des Eingriffs und 
EKG-Detail: $\quad$ Asystolie (ID-Nr. 48), 29-Nov-2014
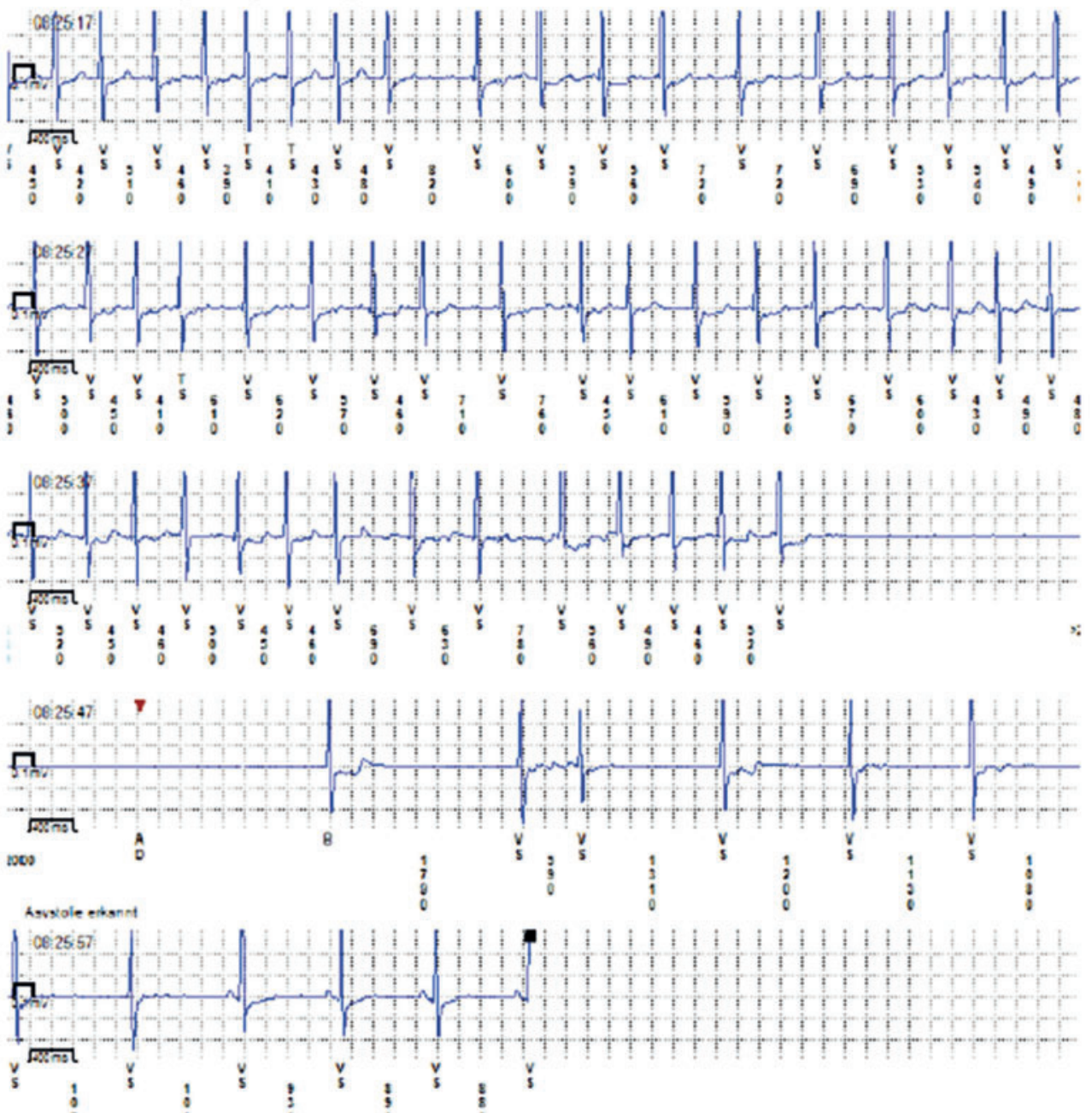

Abb. 12 EKG-Beispiel mit Dokumentation einer präautomatischen Pause nach Terminierung von Vorhofflimmern.

des Systems durch das DRG-System gewährleistet. Für die ambulante Versorgung sind in der Regel Einzelfallkostenanträge und die Zusage des Kostenträgers erforderlich.

\section{Wichtiges in Kürze}

- Herzmonitore leisten einen entscheidenden Beitrag zur Diagnose und Behandlung von Herzrhythmusstörungen.

- Sie helfen dem Arzt, bei unerklärten Synkopen, ungeklärten Schlaganfällen oder bei Palpitationen die Ursache schnellstmöglich aufzudecken und zeitnah die richtige Therapie einzuleiten.

- Das hier vorgestellte Herzmonitor-System der neuesten Generation ist aufgrund seiner Größe und der einfachen praktischen Anwendung für einen ambulanten Eingriff geeignet.

\section{Literatur}

1 Mittal S, Pokushalov E, Romanov A et al. Longterm ECG monitoring using an implantable loop recorder for the detection of atrial fibrillation after cavotricuspid isthmus ablation in patients with atrial flutter. Heart Rhythm 2013; 10: 1598-1604

2 Sciaraffia E, Chen J, Hocini M et al. Use of event recorders and loop recorders in clinical practice: results of the European Heart Rhythm Association Survey. Europace 2014; 16: 1384-1386

3 Roberto M, Martina R, Alice C et al. Additional diagnostic value of implantable loop recorder in patients with initial diagnosis of real or apparent transient loss of consciousness of uncertain origin. Europace 2014; 16: 1226-1230

4 Edvardsson N, Frykman V, van Mechelen R et al. Use of an implantable loop recorder to increase the diagnostic yield in unexplained syncope: results from the PICTURE registry. Europace 2011; 13: 262-269

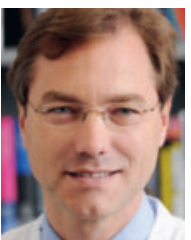

Prof. Dr. med. Thorsten Lewalter ist Direktor der Klinik für Kardiologie am Isar Herz Zentrum in München. Thorsten.Lewalter@ isarklinikum.de

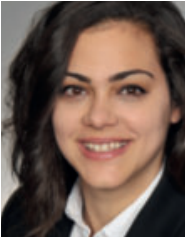

Dr. med. Ilia Koutsouraki ist Assistenzärztin an der Klinik für Kardiologie am Isar Herz Zentrum München. Ilia.Koutsouraki@ isarklinikum.de

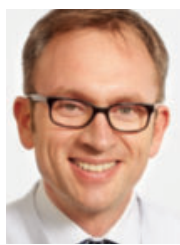

Dr. med. Turgut Brodherr ist leitender Oberarzt Rhythmologie und Ablation am Isar Herz Zentrum in München.

Turgut.Brodherr@ isarklinikum.de

Interessenkonflikt

Die Autoren geben an, dass kein Interessenkonflikt besteht.

DOI 10.1055/s-0041-103932

Dtsch Med Wochenschr 2015; 140: 1286-1289 (c) Georg Thieme Verlag KG . Stuttgart · New York . ISSN 0012-0472 